\title{
Relaxation and the Reentrant Appearance of Phases in a Molecular Monolayer
}

\section{Citation}

Schlossman, M. L., D. K. Schwartz, Peter S. Pershan, E. H. Kawamoto, G. J. Kellogg, and S. Lee. 1991. Relaxation and the reentrant appearance of phases in a molecular monolayer. Physical Review Letters 66(12): 1599-1602.

\section{Published Version}

doi:10.1103/PhysRevLett.66.1599

\section{Permanent link}

http://nrs.harvard.edu/urn-3:HUL.InstRepos:10357549

\section{Terms of Use}

This article was downloaded from Harvard University's DASH repository, and is made available under the terms and conditions applicable to Other Posted Material, as set forth at http:// nrs.harvard.edu/urn-3:HUL.InstRepos:dash.current.terms-of-use\#LAA

\section{Share Your Story}

The Harvard community has made this article openly available.

Please share how this access benefits you. Submit a story.

\section{Accessibility}




\title{
Relaxation and the Reentrant Appearance of Phases in a Molecular Monolayer
}

\author{
M. L. Schlossman, D. K. Schwartz, P. S. Pershan, E. H. Kawamoto, G. J. Kellogg, and S. Lee \\ Department of Physics and Division of Applied Sciences, Harvard University, Cambridge, Massachusetts 02138
}

(Received 27 August 1990)

\begin{abstract}
The structure of monolayers of $\mathrm{CH}_{3}\left(\mathrm{CH}_{2}\right)_{22} \mathrm{COOH}$ on water, along $\sim 21^{\circ} \mathrm{C}$ isotherms between 18 and $35 \AA^{2}$ /molecule, has been studied with glancing-angle $\mathrm{x}$-ray diffraction and specular reflection. Following an incremental area change, in some regions of the isotherm, the diffraction pattern evolves until the surface pressure $\pi$ is relaxing $\lesssim 0.01 \mathrm{dyn} / \mathrm{cmh}$. Reentrant behavior between tilted and nontilted phases is observed. Phase coexistence is indicated by differences between the deposited area/molecule and the area of the $\mathrm{x}$-ray-determined unit cell
\end{abstract}

PACS numbers: $68.10 .-\mathrm{m}, 61.30 . \mathrm{Eb}, 82.65 .-\mathrm{i}$

Langmuir monolayers (LM) on water have been studied as a model for two-dimensional (2D) systems, and for their relevance to biological and technological interfacial phenomena. ${ }^{1-4}$ The most common technique for studying LM has been to measure the surface pressure, $\pi(A)=\gamma_{0}-\gamma(A)$, as a function of the deposited area/ molecule $A$, where $\gamma$ and $\gamma_{0}$ are the surface tensions of the LM and pure water, respectively; however, it has recently become possible to directly measure the structural properties of LM phases by glancing-angle $\mathrm{x}$-ray diffraction. ${ }^{5-13}$

In this Letter we present a detailed study of the $\pi$ - $A$ diagram of tetracosanoic acid $(\mathrm{C} 24)$, at $\sim 20^{\circ} \mathrm{C}$. When the monolayers of tetracosanoic acid are allowed to completely relax both the isotherms and the sequence of phases observed by $x$-ray diffraction are different from those previously published for slightly shorter carboxylic acids. In contrast to the simple phase coexistence that is commonly anticipated, in the "plateau region" we observe reentrant appearance of different phases. In view of the fact that the relaxation times required for stability of these phases are of the order of hours, the nature of the equilibrium in all monolayer studies should be reexamined. ${ }^{4-13}$

The sealed, temperature-controlled Langmuir trough was described previously. ${ }^{14}$ The water, drawn from a Millipore Milli- $Q$ system, was adjusted to $p \mathrm{H} 2(\mathrm{HCl})$. The LM was spread without exposing the trough to air. The surface tension was measured in situ with a resolution of $0.01 \mathrm{dyn} / \mathrm{cm}$ and an accuracy of $\pm 0.5 \mathrm{dyn} / \mathrm{cm}$. The water was judged to be sufficiently clean if $\gamma$, for pure water, changed by $\lesssim 0.2 \mathrm{dyn} / \mathrm{cm}$ over $24 \mathrm{~h}$, and by $\$ 0.05 \mathrm{dyn} / \mathrm{cm}$ when the area was reduced by a factor of 4. When a condensed monolayer was on the surface (i.e., $\pi \geq 3 \mathrm{dyn} / \mathrm{cm}$ ), the change in $\pi$ with time was less than that for clean water.

The C24 ( $>99 \%$ pure, Fluka), spreading solvent (Resi-Analyzed chloroform, Baker), and $\mathrm{HCl}$ (Ultrex, Baker) were used without further purification.

The experiments were performed on beam line X22B at the National Synchrotron Light Source (NSLS), Brookhaven National Laboratory, using a previously described liquid surface reflectometer ${ }^{14-16}$ modified by use of a soller slit (leaves oriented vertically), and a vertically oriented, linear, position-sensitive detector (PSD). The incident angle $\alpha=0.14^{\circ}$ was slightly less than the critical angle for total reflection $\alpha_{c}=0.15^{\circ}$ for a wavelength $\lambda=1.527 \pm 0.006 \AA$. The angular spread of the incident beam was $\Delta \alpha \cong 6 \times 10^{-5}$ rad in the vertical and $\Delta \Phi \cong 1 \times 10^{-3} \mathrm{rad}$ in the horizontal. The horizontal $2 \theta$ resolution was $\Delta 2 \theta \cong 3 \times 10^{-3}$ rad. Scattering from ordered LM produces Bragg rods whose intensity in the $Q_{z}$ direction is a function of both the molecular tilt $\theta_{T}$ and the intrinsic structure factor. The PSD channels were grouped into four bins, resulting in $\Delta Q_{z} \cong 0.06 \AA^{-1}$. For radiation scattered to a vertical angle $\beta$, at an azimuthal angle $2 \theta$, the wave-vector transfer $\mathbf{Q}=\mathbf{Q}_{\text {out }}-\mathbf{Q}_{\text {in }}$; the

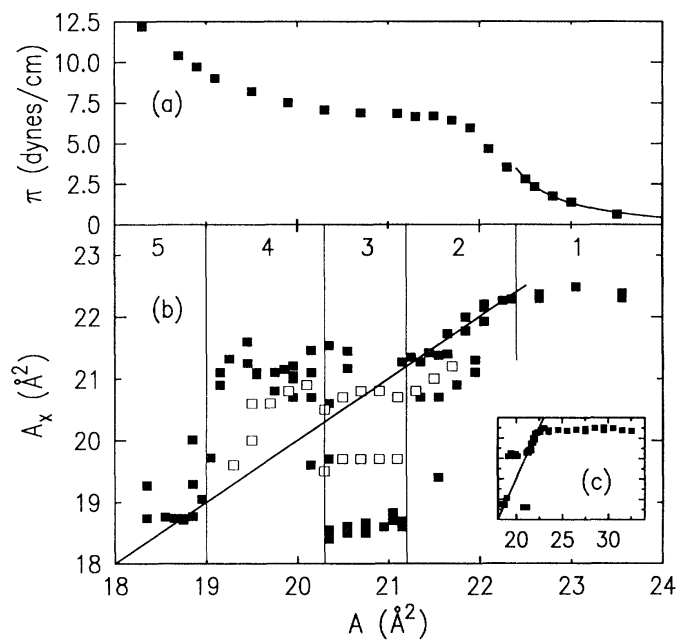

FIG. 1. (a) The solid squares represent a typical isotherm at $T=20.7^{\circ} \mathrm{C}$, taken simultaneously with diffraction measurements; the solid line is discussed at the end of the text. (b) The unit-cell areas $A_{x}$ calculated from the observed diffraction peaks, as a function of the area per molecule deposited in the film $A$. The data shown are a composite of results from six monolayers, in which $A$ has been adjusted to account for systematic errors (i.e., $\Delta A \lesssim \pm 0.5 \AA^{2}$ ); the open squares represent a monolayer compressed more rapidly (see text under region 3). (c) An expanded version of (b) showing the lowdensity region. 
surface normal component $Q_{z}=(2 \pi / \lambda)(\sin \alpha+\sin \beta)$ and the in-plane component

$$
Q_{\perp}=(2 \pi / \lambda)\left[\cos ^{2} \alpha+\cos ^{2} \beta-2(\cos \alpha)(\cos \beta) \cos 2 \theta\right]^{1 / 2} \text {. }
$$

Diffraction measurements of the lowest-order peaks were made on isotherms in the range $T \sim 20-22^{\circ} \mathrm{C}$. Most LM were spread at $\pi \lesssim 3 \mathrm{dyn} / \mathrm{cm}$ and then compressed in steps of 0.1 or $0.2 \AA^{2}$; however, to study lower densities, a few LM were spread at lower $\pi$. For $A \geq 25 \AA^{2}$ complete relaxation was achieved in a few seconds. However, for some parts of the isotherm, diffraction patterns often changed significantly during relaxation periods lasting $\gtrsim 3 \mathrm{~h}$, over which $\Delta \pi \sim 0.1$ $\mathrm{dyn} / \mathrm{cm}$. When $\pi$ stabilized (i.e., $d \pi / d t \lesssim 0.01 \mathrm{dyn} / \mathrm{cm} \mathrm{h}$ ) the diffraction patterns were also stable, and successive steps in $A$ were not taken until both of them were stable. There was no evidence of longer-term drifts that might have been induced by $x$-ray damage to the sample. ${ }^{9,12}$

The solid squares in Fig. 1(a) represent one typical isotherm, taken simultaneously with diffraction measurements. Although diffraction data have been reported at higher pressures than are shown here,,${ }^{6,7,9}$ we find that following further compression the monolayer collapses, with relaxation times of the order of hours, to the maximum pressure shown in Fig. 1(a). The isotherm is divided into five regions whose different structural features are partially characterized by the $2 \mathrm{D}$ powder-diffraction patterns shown in Fig. 2. The detector signal, normalized to the incident intensity, is plotted versus $Q_{\perp}$ for the PSD bin corresponding to the $Q_{z}$ position of the peak. The half-width $\Delta Q_{\perp}$ increases with $Q_{z}$ (or $\beta$ ) as a result of differences in the way the resolution volume intersects the Bragg rod. Most of the peaks in the $I$ and $U$ phases are resolution limited along $Q_{\perp}$, corresponding to $2 \mathrm{D}$ positional correlation lengths $\gtrsim 250 \AA$; peaks in the $F$ phase are usually $\sim 2$ times wider. ${ }^{17}$ There is some $\pi$ dependence [illustrated in Fig. 1(b)] of the peak positions, 2D lattice spacings $(a, b)$, tilt angles $\left(\theta_{T}\right)$, and unit-cell areas $\left(A_{x}\right)$; nevertheless, typical numerical values are listed in Table I for the lattices illustrated in Fig. 2(f). The azimuthal orientations of $\theta_{T}$, for the $I$ and $F$ phases, are indicated by the major axis of the ellipsoids in Fig. 2(f); the $U$ phase is untilted. Some similar structures have been seen for monolayers of different fatty acids ${ }^{7,9}$ and also for smectic- $F$ and $-I$ liquidcrystalline phases. ${ }^{18}$ In addition, the in-plane unit cell of

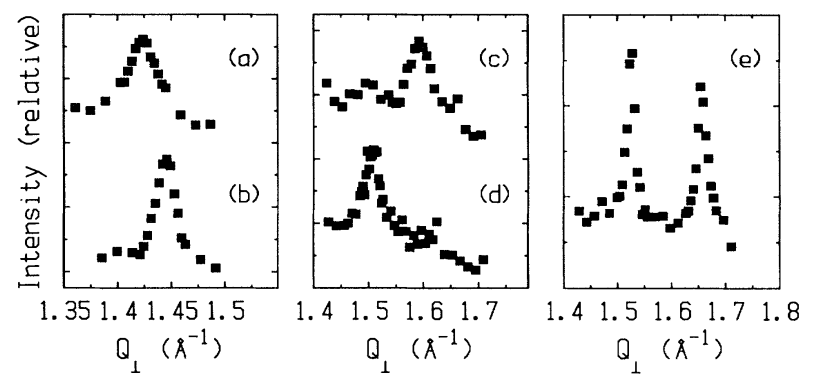

(f)

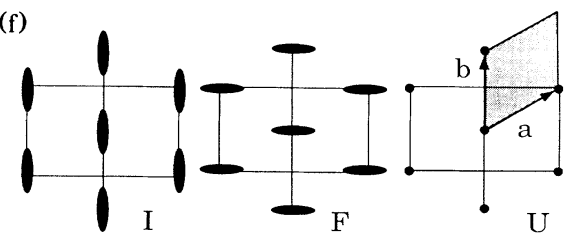

FIG. 2. Scans illustrating diffraction results for the $I$ [(a) $Q_{z}=0.78 \AA^{-1}$, (b) $\left.Q_{z}=0\right], F\left[\right.$ (c) $Q_{z}=0.50 \AA^{-1}$, (d) $Q_{z}$ $\left.=0.24 \AA^{-1}\right]$, and $U\left[(\mathrm{e}) Q_{z} \approx 0\right.$ ] phases. (f) Schematic illustration of the $2 \mathrm{D}$ distorted hexagonal (e.g., rectangular) lattice corresponding to the diffraction results illustrated in (a)-(e). The primitive unit-cell area $A_{x}$ is indicated by the shaded parallelogram. The peak positions and lattice structure for these three data sets are listed in Table I.

the $U$ (and $F$ ) phase is similar to that of the 3D untilted crystalline " $B$ " (and tilted " $C$ ") phase for pure alkanes. ${ }^{19}$

The solid squares in Fig. 1(b) illustrate the variation of $A_{x}$ with $A$, for six different monolayers at $T$ $\cong 20.5^{\circ} \mathrm{C}$. Although there is some slight temperature dependence to the pressure and to the range of area over which a particular phase persists, the sequence of phases is unchanged over the measured temperature range of $20-22^{\circ} \mathrm{C}$. To account for systematic errors (i.e., \pm 0.5 $\AA$ ), the values of $A$ for different monolayers were shifted to ensure that their isotherms overlapped. The solid line, corresponding to $A_{x}=A$, connects the two regions of the film believed to be homogeneous, and is a guide to the eye.

Region 1.- The two characteristic diffraction peaks of the $I$ phase are observed, virtually unchanged, for 35 $\AA^{2} \gtrsim A \gtrsim 22.5 \AA^{2}$. This is similar to results obtained by others on different monolayers and implies that, after spreading, the molecules condense into crystalline or hexatic domains; the remaining $\sim 30 \%$ of the available

TABLE I. The peak positions $\left(Q_{\perp}, Q_{z}\right), 2 \mathrm{D}$ lattice spacings $(a, b)$, unit-cell area $\left(A_{x}\right)$, and tilt from the surface normal $\left(\theta_{T}\right)$ for the data in Figs. 2(a)-2(e).

\begin{tabular}{lcccrrr}
\hline \hline Phase & $\begin{array}{c}A_{x} \\
\left(\AA^{2}\right)\end{array}$ & $Q_{\perp}^{a}, Q_{z}^{a}$ & $Q_{\perp}^{b}, Q_{z}^{b}$ & $a$ & $b$ & $\begin{array}{r}\theta_{T} \\
(\mathrm{deg})\end{array}$ \\
\hline$I$ & 22.3 & $1.445,0.0$ & $1.42,0.78$ & 5.05 & 5.14 & 30 \\
$F$ & 19.4 & $1.60,0.50$ & $1.50,0.24$ & 4.64 & 4.92 & 15 \\
$U$ & 18.5 & $1.66,0.00$ & $1.526,0.0$ & 4.50 & 4.89 & 0 \\
& \pm 0.1 & $\Delta Q_{\perp} \cong \pm 0.005, \Delta Q_{z} \cong \pm 0.05$ & \pm 0.02 & \pm 0.02 & \pm 3 \\
\hline \hline
\end{tabular}


area is occupied by a lower-density phase not detected by diffraction. ${ }^{9,10,13}$ As will be discussed below, it is likely that impurities contribute, in part, to the finite value of $d \pi / d A$ in this region. Because of $\sim 10 \%-50 \%$ stochastic variations in the intensity of the diffraction peaks at $\pm 2 \theta$, we believe that the $x$-ray beam samples $\lesssim 100$ correlated regions. For an illuminated area $\sim 200 \mathrm{~mm}^{2}$ and an incident beam width $\Delta \Phi \sim 0.06^{\circ}$, this implies that the correlated regions are $\gtrsim\left(\left[\left(200 \mathrm{~mm}^{2}\right) / 100\right] 0.06^{\circ} /\right.$ $\left.180^{\circ}\right)^{1 / 2}=26 \mu \mathrm{m}$.

Throughout most of region 1 both $\pi$ and the diffraction spectra stabilize within $\sim 10 \mathrm{~s}$ following compression; however, irregular variations in the specular reflectivity, over several hours, suggest that the monolayer is nonuniform. ${ }^{17}$ At $A \sim 25 \AA^{2}$ the relaxation times abruptly increase to $\sim 10^{3} \mathrm{~s}$, suggesting the onset of a region in which reduction in the free area inhibits the relaxation kinetics.

Region 2.- In addition to the increased relaxation times, region $2\left(21.6 \AA^{2} \lesssim A \lesssim 23.0 \AA^{2}\right)$ differs from region 1 in that (i) $A_{x}$ decreases with decreasing $A$, and (ii) stable specular reflectivity curves are observed. ${ }^{17}$ Both of these observations imply that most of region 2 consists of a uniform monolayer of a single ordered $I$ phase.

Within $\Delta A-0.5 \AA^{2}$ of the boundary between regions 2 and 3 , peaks associated with both $I$ and $F$ phases are observed to coexist. In addition, specular reflection measurements indicate macroscopically coexisting films of different thicknesses. ${ }^{17}$ This is not, however, a simple coexistence because a pure $F$ phase is never observed.

Region 3.- For compression rates $\lesssim 0.05 \AA^{2} / \mathrm{h}$, in the region $20.8 \AA^{2} \lesssim A \lesssim 21.6 \AA^{2}$, diffraction peaks corresponding to the untilted $U$ phase with $A_{x}=18.5 \AA^{2}$ are observed. The area per molecule is essentially identical to the area of $18.45 \AA^{2}$ found in bulk alkanes, indicating that the alkane tail (not the larger carboxyl headgroup) determines the packing. ${ }^{19}$ The fact that $A_{x} / A \lesssim 0.93 \mathrm{im}$ plies that the observed $U$ domains coexist with an undetected lower-density phase. As seen in Fig. 1(b), $A_{x}$ is independent of $A$ in this region. In this same region, but at higher compression rates ( $\geq 0.2 \AA^{2} / \mathrm{h}$ ), coexisting peaks corresponding to only the $I$ and $F$ phases are observed [illustrated by the open squares in Fig. 1(b)]. This leads us to suspect that the unobserved, coexisting phase at low compression rates may be a distorted version of $I$, possibly existing mostly at highly strained grain boundaries. Evidence arguing that the coexistence is not between the $F$ and $U$ phases is the observation that at the boundary between regions 2 and 3, following incremental compression, the $F$ phase is often observed to relax to the $U$.

Region 4. - A major surprise is the reentrant appearance of peaks associated with the $I$ phase for $19.5 \AA^{2} \lesssim$ $A \lesssim 21.0 \AA^{2}$. To demonstrate that this is an equilibrium effect, we show in Fig. 3 scans for $1.42 \AA^{-1} \leq Q_{\perp}$ $\leq 1.57 \AA^{-1}$ at $Q_{z} \cong 0.06 \AA^{-1}$, while the $\mathrm{LM}$ is

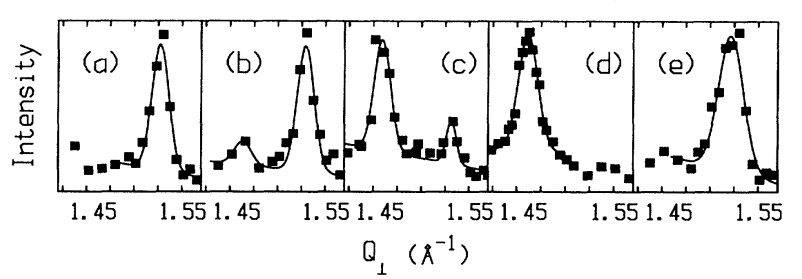

FIG. 3. Illustration of the reversibility of the transition between the $U$ and $I$ phases in regions 3 and $4 ; Q_{\perp}$ scans at $Q_{z}$ $=0.06 \AA^{-1}$; (a) $U$ phase, (b), (c) coexisting $U$ and $I$ phase on compression, (d) pure $I$ phase, and (e) reentrant $U$ phase following expansion. The values of $\pi$ for panels (a)-(e) are 5.90, $6.12,6.15,6.33$, and $6.06 \mathrm{dyn} / \mathrm{cm}$, respectively.

compressed from region 3 to $4[(a)-(d)]$ and then expanded back to 3 [(d) and (e)]. Although there was a small hysteresis in $A\left(\Delta A \lesssim 0.5 \AA^{2}\right)$, as a function of $\pi$, the $U \rightleftarrows I$ transition is reversible.

Below we will speculate on a possible origin for this unusual sequence. The facts that imply that region 4 consists of $I$ domains coexisting with nondiffracting domains of higher density are (1) $A_{x}$, for the observed $I$ domains, is independent of $A$, causing the ratio $A_{x} / A$ $\cong 1.03$, at the boundary between regions 3 and 4 , to rise to 1.13 at the boundary with region 5 , and (2) the specular reflectivity indicates coexisting regions of different thickness. $^{17}$

Region 5.-Finally, for $18.5 \AA^{2} \lesssim A \lesssim 19.5 \AA^{2}$, the phase that appears to be stable, after periods $\sim 5-8 \mathrm{~h}$, is the same $U$ phase that was previously observed in region 3. Although other weak diffraction peaks are occasionally visible, the inhomogeneities that were observed by specular reflectivity in regions 3 and 4 are not observed here. ${ }^{17}$ Furthermore, since $A_{x} \cong A$ and is also comparable to the specific areas of untilted crystalline phases of normal alkanes, ${ }^{19}$ we believe that the monolayer in region 5 is primarily in a single homogeneous $U$ phase.

In summary, the principal result of this Letter is that at sufficiently slow compression rates the sequence of phases for monolayers of tetracosanoic acid, at the water/vapor interface and $T \sim 21^{\circ} \mathrm{C}$, differs from those obtained more rapidly. This has implications for understanding the necessary conditions for equilibrium of compressed monolayers. Second, we have demonstrated that on compression the isolated domains of the island phase of tetracosanoic acid (i.e., region 1) form a relatively homogeneous $I$ phase in which $\Delta A_{x} / \Delta A \sim 1$. Third, regions 3 and 4 , separating the homogeneous $I$ and $U$ phases (regions 2 and 5), are bizarre in that $A_{x} \lessgtr A$. This implies that the diffracting $U$ (or $I$ ) phase in region 3 (or 4 ) covers only a fraction of the surface, the average density in the rest of the surface being lower (or higher).

As illustrated by the solid line in Fig. 1(a), one simple hypothesis regarding the island phase (region 1) is coexistence of $2 \mathrm{D}$ crystalline and gas phases. The line repre- 
sents $\left(\pi-\pi_{0}\right)\left(A-A_{0}\right)=c k_{B} T$, where, for $N$ C24 molecules, the free area available to $c N(c \cong 0.005)$ impurity gas molecules is $N\left(A-A_{0}\right)$ (with $A_{0} \cong 21.9 \AA^{2} \sim A_{x}$ ); $\pi_{0}(\cong-0.5 \mathrm{dyn} / \mathrm{cm})$ accounts for systematic errors in the absolute value of $\pi$. This requires that the gas contain, in addition to $\mathrm{C} 24$, impurity molecules that are insoluble in both the 2D C24 crystalline phase and the 3D aqueous solution. Long-range electric dipole effects, like those discussed by Andelman, Brochard, and Joanny, ${ }^{20}$ might also be important; however, the current data do not justify such speculation.

Although the connection between the bizarre coexistence in regions 3 and 4 and the appearance of reentrant phases in LM is not obvious, reentrant behavior was anticipated theoretically by Nelson. ${ }^{21}$ He predicted that reentrant melting to a hexatic phase could occur in solid films due to quenched random impurities. Since one of the principal differences between the $U$ and $I$ phases is in molecular tilt, it is possible that the paper by Rubinstein, Shraiman, and Nelson ${ }^{22}$ on unbinding of vortices in $X Y$ models may be more relevant. The essential physics in both the Nelson and Rubinstein, Shraiman, and Nelson papers is the minimization of the free energy under constraints associated with local frustration.

If impurities are the source of $d \pi / d A$ in region 1 , then it is natural to suspect that they might also be the source of the random frustration necessary for the reentrance in regions 3 and 4. On the other hand, internal frustrations in the packing of large molecules, ${ }^{18,23-26}$ which are themselves inhomogeneous and asymmetric, could be the source of the reentrant behavior in regions 3 and $4 .^{22}$ It is conceivable that the finite $d \pi / d A$ in region 1 results from electrostatic, or other, repulsion between islands whose maximum dimension is determined by internal frustrations.

We acknowledge conversations with D. Nelson, G. Whitesides, and C. Brown. This work was supported by the National Science Foundation through Grants No. NSF-DMR-88-12855 and No. NSF-DMR-89-20490. Research carried out at the NSLS, Brookhaven National Laboratory, is supported by the Department of Energy, Material Sciences and Division of Chemical Sciences under Contract No. DE-AC02-76CH00016.

' J. D. Swalen et al., Langmuir 3, 932 (1987).

${ }^{2}$ P. S. Pershan, Phys. Today 35, No. 5, 34 (1982).
${ }^{3}$ G. L. Gaines, Jr., Insoluble Monolayers at Liquid-Gas Interfaces (Wiley, New York, 1966).

${ }^{4}$ E. Stenhagen, in Determination of Organic Structures by Physical Methods, edited by E. A. Braude and F. C. Nachod (Academic, New York, 1955).

${ }^{5}$ P. Dutta, J. B. Peng, B. Lin, J. B. Ketterson, M. Prakash, P. Georgopoulos, and S. Ehrlich, Phys. Rev. Lett. 58, 2228 (1987).

${ }^{6}$ S. W. Barton, B. N. Thomas, E. B. Flom, S. A. Rice, B. Lin, J. B. Peng, J. B. Ketterson, and P. Dutta, J. Chem. Phys. 89, 2257 (1988).

${ }^{7}$ R. M. Kenn, C. Böhm, A. M. Bibo, I. R. Peterson, H. Möhwald, K. Kjaer, and J. Als-Nielsen (to be published).

${ }^{8}$ K. Kjaer, J. Als-Nielsen, C. A. Helm, P. Tippmann-Krayer, and H. Möhwald, Thin Solid Films 159, 17 (1988).

${ }^{9}$ B. Lin, M. C. Shih, T. M. Bohanon, G. E. Ice, and P. Dutta, Phys. Rev. Lett. 65, 191 (1990).

${ }^{10}$ S. G. Wolf, E. M. Landau, M. Lahav, L. Leiserowitz, M. Deutsch, K. Kjaer, and J. Als-Nielsen, Thin Solid Films 159, 29 (1988).

${ }^{11}$ S. G. Wolf, L. Leiserowitz, M. Lahav, M. Deutsch, K. Kjaer, and J. Als-Nielsen, Nature (London) 328, 63 (1987).

${ }^{12}$ B. Lin, J. B. Peng, J. B. Ketterson, P. Dutta, B. N. Thomas, J. Buontempo, and S. A. Rice, J. Chem. Phys. 90, 2393 (1989).

${ }^{13}$ D. Jacquemain, S. G. Wolf, F. Leveiller, M. Lahav, L. Leiserowitz, J. Deutsch, K. Kjaer, and J. Als-Nielsen, J. Phys. (Paris), Colloq. 50, C7-29 (1989).

${ }^{14}$ M. L. Schlossman, D. K. Schwartz, E. H. Kawamoto, G. J. Kellogg, P. S. Pershan, B. M. Ocko, M. W. Kim, and T. C. Chung, Mater. Res. Soc. Symp. Proc. 177, 351 (1990).

${ }^{15}$ P. S. Pershan, J. Phys. (Paris), Colloq. 50, C7-1 (1989).

${ }^{16}$ P. S. Pershan, Faraday Discuss. Chem. Soc. 89, 231 (1990).

${ }^{17}$ D. K. Schwartz, M. L. Schlossman, and P. S. Pershan, "X Ray Scattering Studies of Langmuir Monolayers of Tetracosanoic Acid" (unpublished).

${ }^{18}$ P. S. Pershan, Structure of Liquid Crystal Phases (World Scientific, Singapore, 1988).

${ }^{19}$ B. Ewen, G. R. Strobl, and D. Richter, Faraday Discuss. Chem. Soc. 68, 19 (1980).

${ }^{20}$ D. Andelman, F. Brochard, and J.-F. Joanny, J. Chem. Phys. 86, 3673 (1987).

${ }^{21}$ D. R. Nelson, Phys. Rev. B 27, 2902 (1983).

${ }^{22}$ M. Rubinstein, B. Shraiman, and D. R. Nelson, Phys. Rev. B 27, 1800 (1983).

${ }^{23}$ M. Seul and M. J. Sammon, Phys. Rev. Lett. 64, 1903 (1990).

${ }^{24}$ J. M. Carlson and J. P. Sethna, Phys. Rev. A 36, 3359 (1987).

${ }^{25}$ J. F. Sadoc and J. Charvolin, J. Phys. (Paris) 47, 683 (1986).

${ }^{26}$ S. Safran, M. O. Robbins, and S. Garoff, Phys. Rev. A 33, 2186 (1986). 